\title{
Magnetism in Doped Two-Dimensional Honeycomb Structures of III-V Binary Compounds
}

\begin{abstract}
K. ZBERECKI*
Faculty of Physics, Warsaw University of Technology, Koszykowa 75, 00-662 Warsaw, Poland

Using first-principles plane-wave calculations study of magnetic properties of doped two-dimensional honeycomb structures of III-V binary compounds have been conducted for either nonmagnetic dopants or vacancies. Calculations show that all cases where magnetic moment is non-zero, are energetically more favorable. For such cases band structure and density of states were calculated and analyzed in detail. The possible applications of these structures were also discussed.
\end{abstract}

PACS: 73.22.-f, 75.50.Pp, 75.50.Dd, 75.70.Ak

\section{Introduction}

Since its discovery in 2004 graphene [1] draws much attention beacuse of unique features of this two-dimensional system. Graphene is composed of a $s p^{2}$ -bonded carbon atoms forming honeycomb structure. It has very interesting electronic structure with characteristic, linear energy dispersion near $K$ point of the Brillouin zone (see e.g. [2]).

Shortly after, experimental techniques allowed fabrication of other new two-dimensional materials, like BN and $\mathrm{MoS}_{2}$ honeycomb structures [3] or ZnO monolayers [4]. Also many these hypothetical structures constructed from silanene $(2 \mathrm{D} \mathrm{Si})$ and germanene $(2 \mathrm{D} \mathrm{Ge})$ [5, 6], III-V compounds [7], $\mathrm{SiC}$ [8] or $\mathrm{ZnO}$ [9] have been studied theoretically.

On the other hand, graphene and other nanoscale materials are recognized as future building blocks of new electronics technologies [10], including spintronics. In the case of low (one- and two-) dimensional structures problem arises because of famous Mermin-Wagner theorem [11], which prevents ferro- or antiferromagnetic order to occur in finite temperatures. This started the theoretical and experimental search for magnetism in graphene and other structures. According to works of Yazyev [12] single atom-defects can induce ferromagnetism in graphene based materials. Magnetic order arises as an effect of presence of single-atom defects in combination with a sublattice discriminating mechanism, in agreement with Lieb's theorem [13]. Based on these findings several theoretical studies have been conducted in search for magnetism in low-dimensional structures either for graphene and $\mathrm{BN}$ [14] or other structures [8].

In this paper influence of local defects on magnetic structure of two-dimensional honeycomb structures of GaN and AlN have been analysed by means of $a b i n i-$ tio calculations, since bulk versions of these compounds are very important semiconductors in todays electronics. Despite of the fact that neither of them have been yet synthesized, calculated cohesion energies [7] suggest that

* e-mail: zberecki@if.pw.edu.pl such structures would be stable and their experimental procurement is highly probable.

\section{Computational details}

To investigate magnetic properties of GaN and AlN honeycomb structures a series of $a b$ initio calculations have been conducted with use of DFT VASP code $[15,16]$. The exchange-correlation potential has been approximated by generalized gradient approximation (GGA) using PW91 functional [17]. Kinetic energy cutoff of $500 \mathrm{eV}$ for plane-wave basis set has been used. Supercells of size $3 \times 3 \times 1$ have been checked to be large enough to prevent defects interaction with its periodic image. In all cases for self-consistent structure optimizations, the Brillouin zone (BZ) was sampled by $20 \times 20 \times 1$ special $k$ points. All structures have been optimized unless the Feynman-Hellman forces acting on each atom become smaller than $10^{-4} \mathrm{eV} / \AA$. A vacuum spacing of $12 \AA$ was applied to hinder the interactions between monolayers in adjacent supercells.

\section{Results}

As mentioned, non-magnetic honeycomb sheets can attain spin polarized states due to presence of local defects. In this work two kinds of defects have been analysed vacancies and substitutions by alkali metal elements and alkaline earth metal elements to check whether vacancy or substitution by few-valence electron atom can lead to non-zero magnetic moment.

For both compounds a vacancy was generated first by removing a single atom, $\mathrm{Al}, \mathrm{Ga}$ or $\mathrm{N}$ from each supercell, then the atomic structure was optimized. In both cases, structures with single $\mathrm{N}$ vacancy are non-magnetic, while $\mathrm{Al}$ or $\mathrm{Ga}$ vacancies induce non-zero magnetic moment, equal to $3.00 \mu_{\mathrm{B}}$.

Figure 1 shows density of states (DoS) for spin-polarized Al-vacant AlN and Ga-vacant GaN, on which difference between majority spin (up) and minority spin $(\mathrm{dn})$ in the vicinity of the Fermi level can be observed. This is the main source of non-zero magnetic moment. Analysis of calculated partial magnetisation shows that 


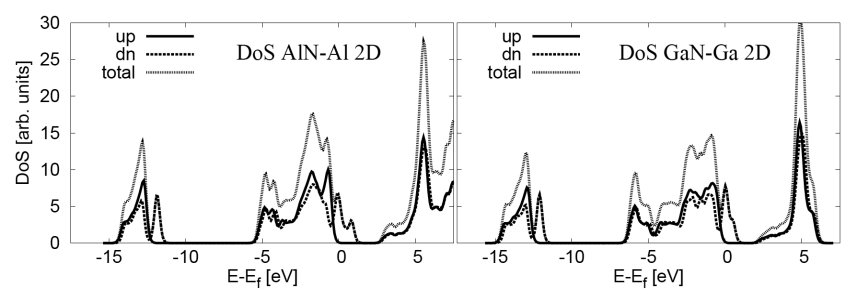

Fig. 1. Density of states of Al-vacant AlN and Ga-vacant GaN. See text for details.

almost whole magnetic moment is situated on $p$-states of $\mathrm{N}$ atoms located in the area of vacancy. This is in full agreement with previous studies of vacancies in $\mathrm{SiC}$ [8]. For both cases with non-zero magnetic moment, the total energy for spin-polarized case has been lowered by $0.33 \mathrm{eV}$ and $0.24 \mathrm{eV}$, respectively, for Al-vacant AlN and Ga-vacant GaN.

In the case of substitution the procedure was as follows. For both compounds various single foreign atoms have been substituted, then structure has been optimized. In the case of AlN, Al has been substituted by Na, Mg, K and $\mathrm{Ca}$ atoms, in the case of GaN, Ga has been substituted by $\mathrm{K}$ and $\mathrm{Ca}$ atoms. Only for the case of Naand K-doped AlN magnetic moment has been non-zero, equal to $1.95 \mu_{\mathrm{B}}$ and $1.70 \mu_{\mathrm{B}}$, respectively. Calculated total energy shows that in both cases spin-polarized state is more energetically favorable, by $0.06 \mathrm{eV}$ and $0.04 \mathrm{eV}$.

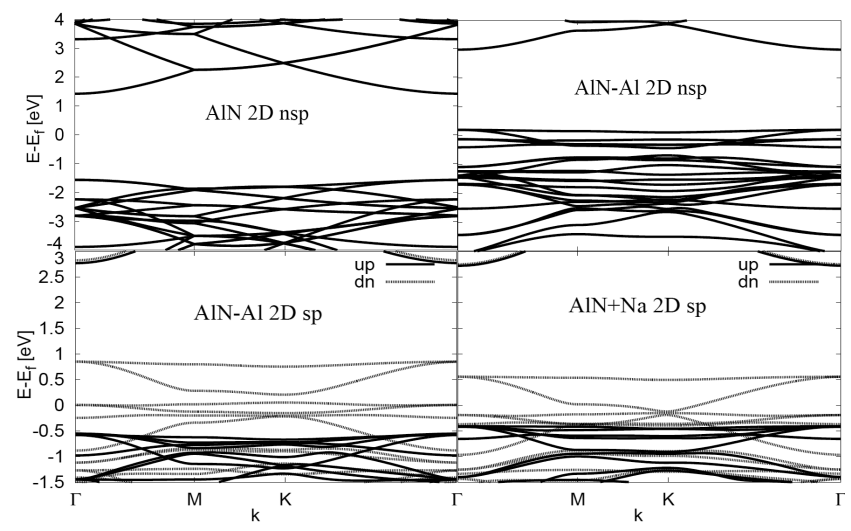

Fig. 2. Band structure for Al-vacant and Na-doped AlN. See text for details.

Figure 2 shows calculated band structure of Al-vacant AlN and Na-doped AlN. Top figures show band structure of Al-vacant AlN (right) and (left) undoped one (which is a semiconductor with band gap equal to $3.10 \mathrm{eV}$, calculated within GGA) in spin-unpolarized case. One can see that vacant structure is metallic. Bottom left figure shows band structure of $\mathrm{AlN}-\mathrm{Al}$ in the spin-polarized case, where can be observed quite large splitting of these bands between spin up and down bands. Right figure shows analogous plot for $\mathrm{AlN}+\mathrm{Na}$ case. As one can observe, these two are quite similar which is not surprising, since $\mathrm{Na}$ atom has only one valence electron. So in both cases - vacant structure and alkali metal element doped - the mechanism of formation of magnetic moment is similar.

\section{Conclusions}

$A b$ initio calculations have been conducted for vacancy and substitution defects in honeycomb AlN and GaN compounds. Calculations show that in both compounds vacancy of $\mathrm{Al}$ or $\mathrm{Ga}$ gives magnetic moment of $3.00 \mu_{\mathrm{B}}$, which is interesting conclusion from application point of view. On the other hand, substitution of $\mathrm{Al}$ or $\mathrm{Ga}$ by alkali metal elements can give lower, but non-zero magnetic moments. This suggests that, from application point of view, AlN can be magnetized without doping.

\section{Acknowledgments}

Numerical calculations were performed at the Interdisciplinary Centre for Mathematical and Computational Modelling (ICM) at Warsaw University.

\section{References}

[1] K.S. Novoselov, A.K. Geim, S.V. Morozov, D. Jiang, Y. Zhang, S.V. Dubonos, I.V. Grigorieva, A.A. Firsov, Science 306, 666 (2004).

[2] A.H. Castro Neto, F. Guinea, N.M.R. Peres, K.S. Novoselov, A.K. Geim, Rev. Mod. Phys. 81, 109 (2009).

[3] K.S. Novoselov, D. Jiang, F. Schedin, T.J. Booth, V.V. Khotkevich, S.V. Morozov, A.K. Geim, Proc. Natl. Acad. Sci. USA 102, 10451 (2005).

[4] C. Tusche, H.L. Meyerheim, J. Kirschner, Phys. Rev. Lett. 99, 026102 (2007).

[5] S. Lebegue, O. Eriksson, Phys. Rev. B 79, 115409 (2009).

[6] S. Cahangirov, M. Topsakal, E. Akturk, H. Sahin, S. Ciraci, Phys. Rev. Lett. 102, 236804 (2009).

[7] H. Sahin, S. Cahangirov, M. Topsakal, E. Bekaroglu, E. Akturk, R. T. Senger, S. Ciraci, Phys. Rev. B 80, 155453 (2009).

[8] E. Bakaroglu, M. Topsakal, S. Cahangirov, S. Ciraci, Phys. Rev. B 81, 075433 (2010).

[9] M. Topsakal, S. Cahangirov, E. Bekaroglu, S. Ciraci, Phys. Rev. B 80, 235119 (2009).

[10] P. Avouris, Z. Chen, V. Perebeinos, Natur. Nanotech. 2, 605 (2007).

[11] N.D. Mermin, H. Wagner, Phys. Rev. Lett. 17, 1133 (1966).

[12] O. Yazyev, Phys. Rev. Lett. 101, 037203 (2008).

[13] E.H. Lieb, Phys. Rev. Lett. 62, 1201 (1989); 62, 1927(E) (1989).

[14] C. Ataca, S. Ciraci, Phys. Rev. B 82, 165402 (2010).

[15] G. Kresse, J. Hafner, Phys. Rev. B 47, 558 (1993).

[16] G. Kresse, J. Furthmuller, Phys. Rev. B 54, 11169 (1996).

[17] J.P. Perdew, J.A. Chevary, S.H. Vosko, K.A. Jackson, M.R. Pederson, D.J. Singh, C. Fiolhais, Phys. Rev. B 46, 6671 (1992). 